\title{
Estudio preliminar morfológico y morfométrico de encéfalo del pez tiburoncito, Ariopsis seemanni, (Pisces: Ariidae)
}

\author{
Claudia Londoño ${ }^{1}$, Hernán Hurtado Giraldo ${ }^{2,3}$ \\ ${ }^{1}$ Estudiante programa de Biología Aplicada, ${ }^{2}$ Director Grupo Ictiología, programa de Biología Aplicada, Facultad de Ciencias, \\ Universidad Militar Nueva Granada, Cajicá. Colombia \\ "hernan.hurtado@unimilitar.edu.co
}

Recibido: 24-05-2010; Aceptado: 12-07-2010

\begin{abstract}
Resumen
Objetivo. Describir morfológica y morfométricamente el cerebro de tiburoncito Ariopsis seemanni. Materiales y métodos. Se trabajó con 10 ejemplares juveniles de tiburoncito los cuales fueron procesados con las normas éticas para el manejo de peces. Posteriormente se tomaron medidas de longitud total y estándar $(\mathrm{cm})$ y de peso $(\mathrm{g})$ de cada uno de los individuos, que se fijaron en formaldehido al $4 \%$. Una vez retirados los encéfalos se realizó la identificación y medición de los diferentes lóbulos indicando su ubicación, morfología, longitud y área a través de imágenes laterales, dorsales y ventrales digitalizadas, las cuales fueron medidas con el programa Scion Image. Se calcularon promedios y desviaciones estándar, y se obtuvo la equivalencia porcentual de cada estructura en relación con la totalidad del encéfalo. Estos datos se relacionaron posteriormente con los hábitos de la especie. Resultados. Se identificaron los bulbos olfativos, hemisferios telencefálicos, lóbulos ópticos, cerebelo, entre otros. De los nervios craneales, únicamente se observó el nervio óptico. La estructura más grande del encéfalo es el cerebelo $\left(44 \pm 1,2 \%\right.$ y área $\left.0,23 \pm 0,03 \mathrm{~cm}^{2}\right)$, seguido por los hemisferios telencefálicos $(28 \pm 0,57 \%$ y área $0,13 \pm 0,02$ $\mathrm{cm}^{2}$ ). Conclusiones. La morfología del encéfalo de A. seemanni presentó el patrón general de teleósteos del orden Siluriforme, contando con el cerebelo como la estructura más grande. Según la morfología y morfometría vista en el encéfalo, se podría postular que $A$ seemanni emplea sobre todo la vista y el gusto para explorar el ambiente.
\end{abstract}

Palabras clave: teleósteos, morfología cerebral, sistema sensorial, neuroecología, Ariopsis seemanni.

\begin{abstract}
Preliminary morphologic and morphometric study of the encephalon of the tiburoncito Ariopsis seemanni (Pisces: Ariidae) Objective. To describe morphologically and morphometrically the encephalon of Ariopsis seemanni. Materials and methods. We worked with 10 juvenile specimens which were processed according to the ethical standards for handling fish. Measurements of the total and standard length $(\mathrm{cm})$ and weight $(\mathrm{g})$ were taken for each individual. Specimens were fixed in $4 \%$ formaldehyde. The encephalon was removed, the different regions were identified and the measurement of the different lobes was done, indicating their location, morphology, length and area using lateral, dorsal and ventral digitalized images that were measured with the Scion Image program. We calculated means and standard deviations, and obtained the percentage equivalence of each structure in relation to the entire encephalon. These data were then related to the habits of the species. Results. We identified the olfactory bulbs, telencephalic hemispheres, optic lobes, cerebellum, among others. Of the cranial nerves, only the optic nerve was observed. The largest structure of the encephalon is the cerebellum ( $44 \pm 1.2 \%$ and $0.23 \pm 0.03 \mathrm{~cm}^{2}$ area), followed by the telencephalic hemispheres $\left(28 \pm 0.57 \%\right.$ and $0.13 \pm 0.02 \mathrm{~cm}^{2}$ area). Conclusions. The encephalon morphology of A. seemanni presented the general pattern of teleosts of the order Siluriformes, with the cerebellum as the largest structure of the encephalon. Based on the morphology and morphometry observed in the encephalon, we suggest that A. seemanni uses primarily vision and taste to explore the environment.
\end{abstract}

Key words: teleost, cerebral morphology, sensory system, neuroecolgy, Ariopsis seemanni. 


\begin{abstract}
Resumo
Estudo preliminar morfológico e morfométrico do encéfalo do Peixe Gato Tete, Ariopsis seemanni (Pisces: Ariidae). Objetivo. Descrever os aspectos morfológicos e morfométricos do cérebro do peixe gato tete, Ariopsis seemanni. Materiais e métodos. A pesquisa foi realizada com 10 destes peixes, que foram tratados com as normas éticas para a manipulação de peixes. Posteriormente foram registrados o comprimento total e padrão $(\mathrm{cm})$ e o peso $(\mathrm{g})$ de cada um dos indivíduos, os quais foram fixados em formol $4 \%$. Depois de retirar os encéfalos foi realizada a identificação e medição dos diferentes lóbulos, indicando sua localização, morfologia, o comprimento e a área através de imagens laterais, dorsais e ventrais digitalizadas, que foram medidas com o programa Scion Image. Foram calculados a média e o desvio padrão, e obteve-se a equivalência percentual de cada estrutura em relação a todo o encéfalo. Estes dados foram correlacionados com os hábitos da espécie. Resultados. Foram identificados os bulbos olfatórios, hemisférios telencefálico, lobos ópticos, cerebelo, entre outros. Dos nervos cranianos, apenas o nervo ótico foi observado. A maior estrutura do encéfalo é o cerebelo $\left(44 \pm 1,2 \%\right.$ e $0,23 \pm 0,03 \mathrm{~cm}^{2}$ de área), seguido pelos hemisférios telencefálicos $\left(28 \pm 0,57 \%\right.$ e área $\left.0,13 \pm 0,02 \mathrm{~cm}^{2}\right)$. Conclusões. A morfologia do encéfalo de $A$. seemanni apresentou o padrão geral de teleósteos da ordem Siluriformes, com o cerebelo como a maior estrutura. De acordo com a morfologia e morfometria vista no encéfalo, pode-se postular que um A seemanni usa principalmente a visão e o gosto para explorar o ambiente.
\end{abstract}

Palavras-chave: teleósteo, morfologia cerebral, sistema sensorial, neuroecologia, Ariopsis seemanni.

\section{Introducción}

Ariopsis seemanni pertenece a la familia Ariidae, orden Siluriformes (bagres), los cuales se caracterizan por no tener escamas sobre el cuerpo, encontrándose revestido de piel gruesa o cubierto parcial o totalmente por placas óseas, así mismo presenta barbas que tienen una función sensorial (1). A. seemanni posee una forma alargada, ocho aletas y puede alcanzar un tamaño hasta de $60 \mathrm{~cm}$ (2). Se encuentra en cauces fluviales y estuarios con comunicación con el Océano Pacífico (3). Su medio natural está enmarcado por aguas salobres o saladas con un $\mathrm{pH}$ que oscila entre los 6.8 y 8.0, y una temperatura entre los 22 y $26{ }^{\circ} \mathrm{C}$ (3). Esta especie tiene interés comercial debido a su atractivo físico, lo que lo hace un pez ideal para ornamentar acuarios (2). La relación especie-hábitat es de gran importancia y se encuentra muy marcada en términos de modificaciones en el sistema sensorial en busca de incrementar la eficiencia en la interacción con el medio (4). Kihslinger y Nevitt (2005) aseguraron que el ambiente en el que se ha desarrollado el cerebro de los teleósteos influencia el comportamiento individual y las preferencias de hábitat en la vida del adulto (5). El encéfalo es el principal controlador de muchos procesos fisiológicos, los cuales están mediados por estímulos que son percibidos por el individuo a través de estructuras sensibles como los ojos, la piel, las barbillas, entre otros (6).

Ahora bien, a pesar de la importancia que tiene el encéfalo, estudios sobre la relación entre la morfología de esta estructura y su relación con el hábitat no se han realizado en A. seemanni. Conocer esta relación aporta información acerca de las probables respuestas de la especie a diferentes estímulos a los que pueda ser sometido en su medio natural y/o en cultivo. Para esto es importante tener en cuenta que el encéfalo se divide en tres diferentes partes, cerebro anterior, medio y posterior (7). El segmento anterior comprende telencéfalo y diencéfalo los cuales a su vez poseen bulbos olfativos, hemisferios telencefálicos, el tracto olfativo, tálamo dorsal, tálamo ventral e hipotálamo $(8,9)$. El segmento medio, que posee un tectum óptico dorsal y un tegumento en la parte ventral (10). El segmento posterior que incluye el cerebelo, el mielencéfalo y la medula oblongada, la cual se encuentra dividida en los lóbulos vagales y faciales (7-9).

\section{Materiales y métodos}

\section{Material biológico}

Para este proyecto se trabajó con 10 ejemplares juveniles (Figura 1. No se determinó el sexo de los especímenes) de tiburoncito, proporcionados por el laboratorio de Acuicultura de la Universidad Militar Nueva Granada. Los peces utilizados tenían $10,5 \pm 1,1 \mathrm{~cm}$ de longitud total, 8,5 $\pm 0,9 \mathrm{~cm}$ de longitud estándar y un peso de $11,92 \pm 4,1 \mathrm{~g}$. Los peces fueron mantenidos en un sistema de recirculación cerrado de agua con capacidad de $250 \mathrm{~L}$ y alimentados ad libitum con Truchina al $45 \%$ p/p de proteína.

\section{Procesamiento de las muestras}

Los individuos fueron anestesiados con Benzocaina $\left(0,1 \mathrm{gL}^{-1}\right)$, sumergiéndolos en un baño de esta solución. Una vez los individuos perdieron el eje de nado, y los movimientos bucal y opercular, se procedió a realizar un corte transversal en la médula espinal para sacrificarlos, teniendo en cuenta las normas éticas para el manejo de peces (11). Luego, se realizó una incisión craneal con el 


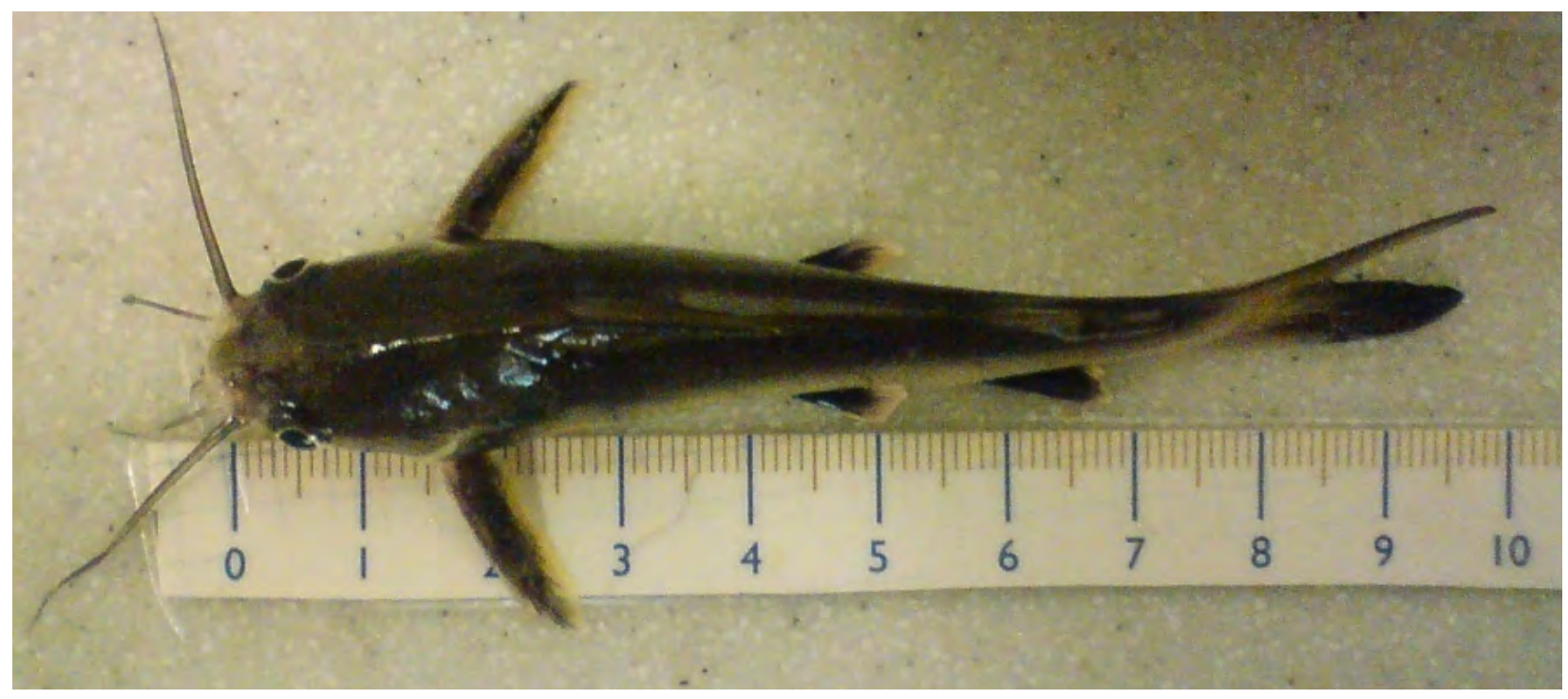

Figura 1. Ejemplar juvenil de tiburoncito. Escala en $\mathrm{cm}$.

fin de permitir que el fijador entrara al encéfalo. Los individuos se introdujeron en formaldehido al $4 \% \mathrm{v} / \mathrm{v}$ (amortiguado, pH 7,2 $\pm 0,2$ ) y fueron mantenidos en este fijador por una semana. Se procedió a realizar la disección bajo estereoscopio (ZEISS) para obtener el encéfalo. Una vez extraídos, se mantuvieron en formaldehido al $4 \% \mathrm{v} / \mathrm{v}$.

\section{Descripción morfológica}

Se realizó la identificación de las diferentes estructuras indicando su ubicación y morfología, a partir de atlas ya publicados como el de Medaka (12), la anguila japonesa (13), así como de otros teleósteos $(14,15)$, y de la experiencia previa del grupo de ictiología de la Universidad Militar Nueva Granada con otras especies de peces (Piaractus brachypomus, Eremophylus mutissi, Paracheirodon axelrodi y Pimelodus pictus). Para esta identificación se tomaron fotos digitales laterales, dorsales y ventrales del encéfalo.

\section{Descripción morfométrica}

Con las imágenes digitales se tomaron medidas de longitud total del encéfalo y de algunos lóbulos (bulbos olfativos, hemisferios telencefálicos, lóbulos ópticos, cerebelo, crista cerebelar y lóbulos vagales, faciales e inferiores). Las medidas de longitud se realizaron sobre las imágenes laterales del encéfalo, ya que estas son las que permiten obtener la mayor longitud de cada estructura, y se siguió la metodología propuesta por Marchetti y Nevitt (2003). (16).

Así mismo, se tomaron medidas de área de bulbos olfativos, hemisferios telencefálicos, lóbulos ópticos, cerebelo, crista cerebelar, hipófisis y lóbulos vagales, faciales e inferiores. Las medidas de área se realizaron sobre las imágenes dorsales para los lóbulos olfativos, hemisferios telencefálicos, cerebelo, crista cerebelar y lóbulos vagales y faciales, imágenes ventrales para los lóbulos inferiores e hipófisis e imágenes laterales para los lóbulos ópticos. Las medidas se tomaron a partir de las imágenes que ofrecen mayor área expuesta.

Todas las medidas de área y longitud se tomaron con el programa Scion Image-Release Beta 4.0.2 2009, el cual es de libre distribución en Internet (www.scioncorp.com).

\section{Análisis de datos}

Una vez obtenidos los datos de área y longitud, se calculó la equivalencia porcentual de la longitud de cada estructura evaluada en relación con la longitud total del encéfalo, posteriormente se calcularon promedios y desviaciones estándar. En la longitud total del encéfalo no se tuvo en cuenta la longitud de los tractos olfativos pues estos son considerados una estructura de conducción de información entre los bulbos olfativos y los hemisferios 
telencefálicos y no como una estructura de integración (8 y 9). En cuanto al área, se calcularon promedios y desviaciones estándar a cada una de las estructuras evaluadas

Estos datos se relacionaron posteriormente con los hábitos de la especie, teniendo en cuenta el hábitat descrito para $A$. seemanni por Froese y Pauly, 2008 (2) y otros estudios similares realizados en teleósteos en donde se revela que la morfología del encéfalo se relaciona con parámetros comportamentales y ecológicos $(15,17,18)$.

\section{Resultados}

\section{Morfología y morfometría Bulbos Olfativos}

Estos se observan en la parte anterior del encéfalo junto a las narinas: tienen forma redondeada y se encuentran conectados a los hemisferios telencefálicos a través del tracto olfativo (Figuras 2, 3 y 4). Poseen un área promedio de $0,042 \pm 0,01 \mathrm{~cm}^{2}$ y equivalen al $9,35 \pm 1,36 \%$ de la longitud total (Tabla 1).

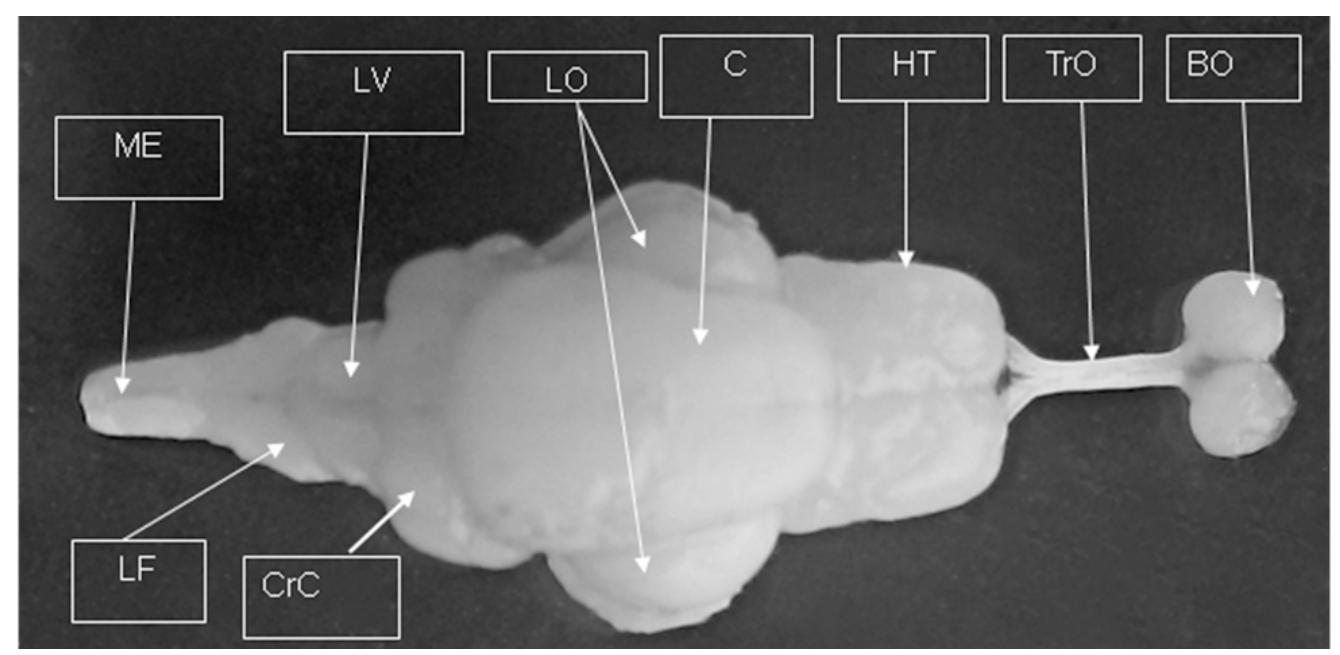

Figura 2. Vista dorsal del encéfalo de A. seemanni. BO; Bulbos Olfativos, TrO; Tractos Olfativos, HT; Hemisferios Telencefálicos, LO; Lóbulos Ópticos, C; Cerebelo, CrC; Crista Cerebelaris, LV; Lóbulos Vagales, LF; Lóbulos Faciales, ME; Médula Espinal. 7x.

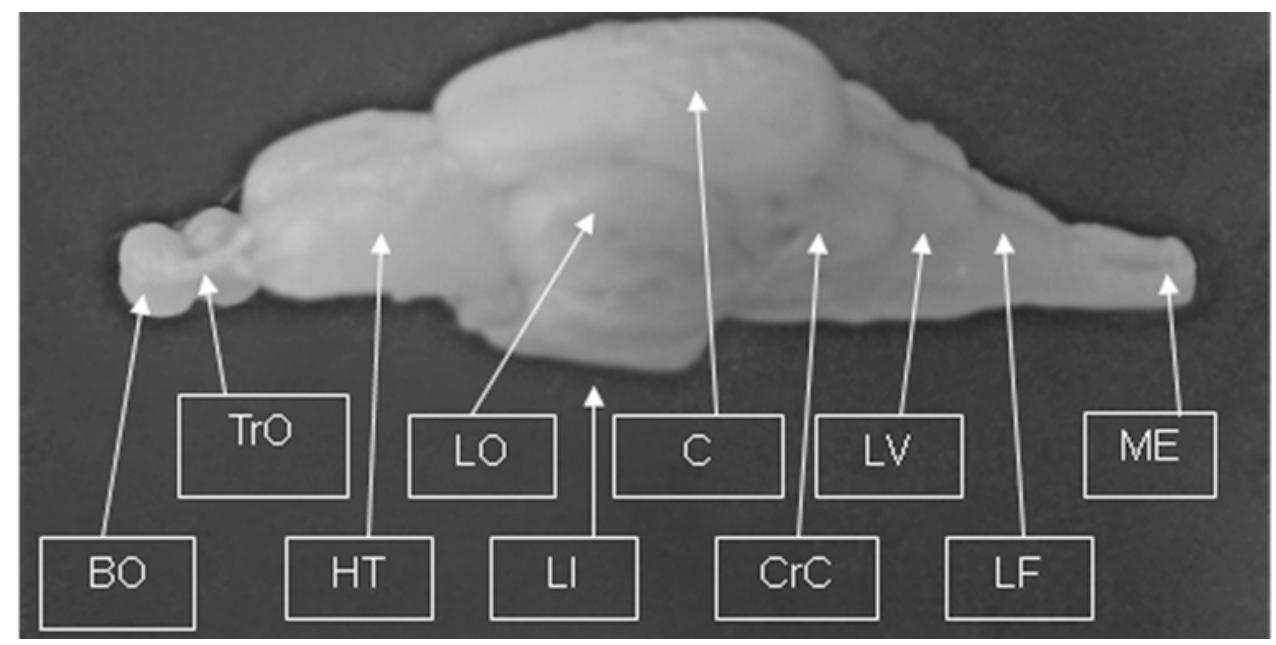

Figura 3. Vista lateral del encéfalo de A. seemanni. BO; Bulbos Olfatorios, TrO; Tractos Olfatorios, HT; Hemisferios Telencefálicos, LO; Lóbulos Ópticos, LI; Lóbulos Inferiores, C; Cerebelo, CrC; Crista Cerebelaris, LV; Lóbulos Vagales, LF; Lóbulos Faciales, ME; Médula Espinal. 7x. 


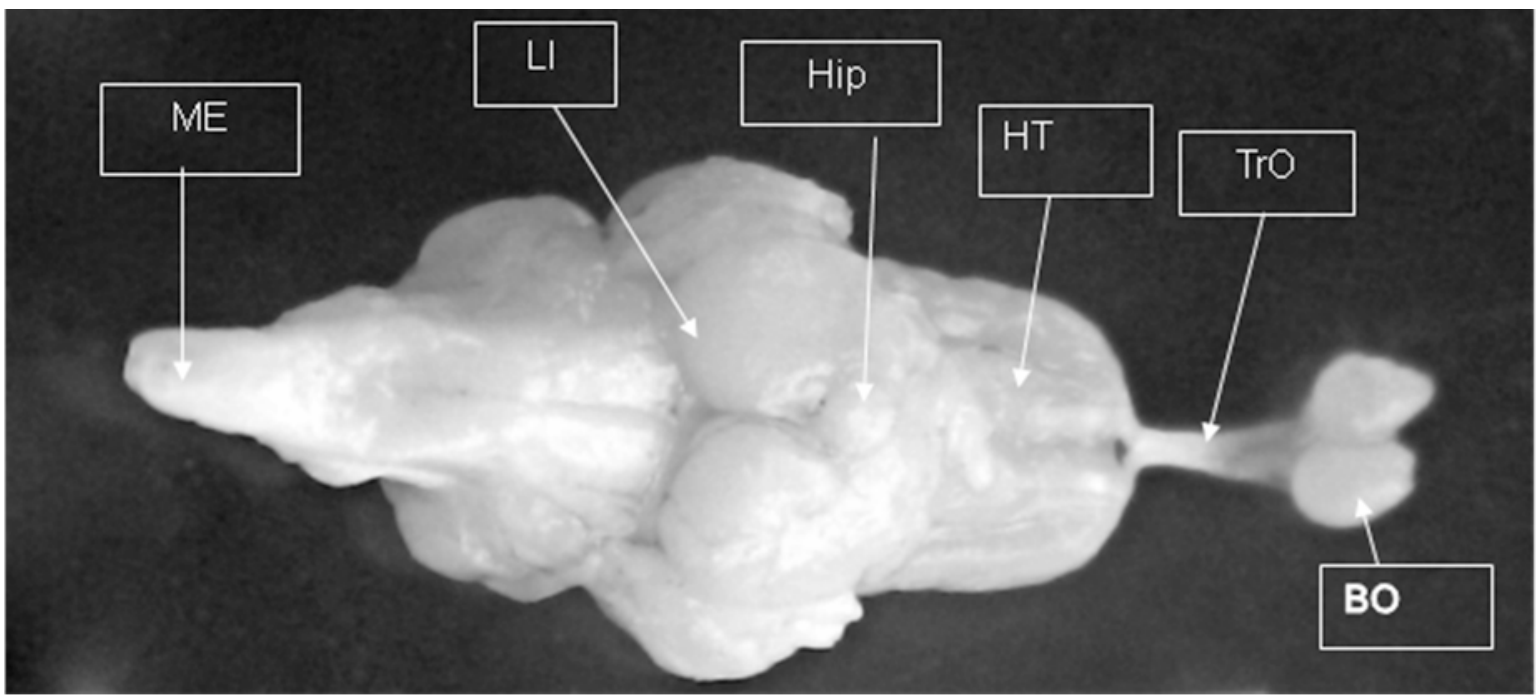

Figura 4. Vista ventral del encéfalo de A. seemanni. BO; Bulbos Olfatorios, TrO; Tractos Olfatorios, HT; Hemisferios Telencefálicos, LI; Lóbulos Inferiores, Hip; Hipófisis, ME; Médula Espinal. 7x.

\section{Tractos Olfativos}

Son estructuras cilíndricas, alargadas y delgadas. Conectan a los bulbos olfativos con los hemisferios telencefálicos en sentido antero posterior (Figuras 2, 3 y 4). Poseen una longitud promedio igual a $0,31 \pm 0,17 \mathrm{~cm}$ (Tabla 1).

\section{Hemisferios Telencefálicos}

Son estructuras con forma ovalada, casi cilíndrica, pareadas (Figuras 2, 3 y 4). En promedio equivalen al 28.15 \pm 0.57
$\%$ de la longitud total y el área promedio es de $0.13 \pm 0.02$ $\mathrm{cm}^{2}$ (Tabla 1).

\section{Lóbulos Ópticos}

Se ubican caudal a los hemisferios telencefálicos (Figura 2); tienen forma ovalada y están separados uno del otro. Se conectan a los ojos a través de los nervios ópticos los cuales se encuentran en la parte ventral del cerebro. Equivalen en promedio al $25.98 \pm 0.57 \%$ de la longitud total y poseen un área promedio de $0.072 \pm 0.01 \mathrm{~cm}^{2}$ (Tabla 1$)$.

Tabla 1. Morfometría del encéfalo del pez tiburoncito, A. seemani. ND: no determinado

\begin{tabular}{lccc}
\hline Estructura & Longitud $(\mathbf{c m})$ & $\begin{array}{c}\text { Equivalencia } \\
\text { porcentual } \\
\text { en longitud }\end{array}$ & Área $\left.\mathbf{( c m}^{\mathbf{2}}\right)$ \\
\hline Cerebro & $1,39 \pm 0,10$ & 100 & $\mathrm{ND}$ \\
Bulbos Olfativos & $0,13 \pm 0,03$ & $9,35 \pm 1,36$ & $0,042 \pm 0,01$ \\
Hemisferios Telencefálicos & $0,39 \pm 0,03$ & $28,15 \pm 0,57$ & $0,13 \pm 0,02$ \\
Lóbulos Ópticos & $0,36 \pm 0,03$ & $25,98 \pm 0,57$ & $0,072 \pm 0,01$ \\
Cerebelo & $0,61 \pm 0,05$ & $44 \pm 1,28$ & $0,23 \pm 0,03$ \\
Crista Cerebelaris & $0,24 \pm 0,01$ & $17,10 \pm 0,86$ & $0,07 \pm 0,01$ \\
Lóbulos vagales & $0,11 \pm 0,01$ & $7,70 \pm 0,59$ & $0,02 \pm 0,01$ \\
Lóbulos Faciales & $0,11 \pm 0,02$ & $8,18 \pm 0,80$ & $0,03 \pm 0,01$ \\
Lóbulos Inferiores & $0,37 \pm 0,05$ & $26,64 \pm 1,67$ & $0,15 \pm 0,02$ \\
Tractos olfativos & $0,31 \pm 0,14$ & $\mathrm{ND}$ & $\mathrm{ND}$ \\
\hline
\end{tabular}




\section{Cerebelo}

Está compuesto por un solo lóbulo y tiene forma ovalada. Se encuentra sobrepuesto a una parte de los lóbulos ópticos (Figura 2). En promedio equivale al $44 \pm 1,28 \%$ de la longitud total y presenta un área promedio de $0,23 \pm 0,03$ $\mathrm{cm}^{2}$ (Tabla 1).

\section{Crista Cerebelaris}

Tal como lo muestra la figura 1, la crista cuenta con dos lóbulos yuxtapuestos de forma ovalada (Figura 2 y 3), los cuales se encuentran detrás del cerebelo y cuenta con el $17,10 \pm 0,86 \%$ de la longitud total y posee un área promedio de $0,07 \pm 0,01 \mathrm{~cm}^{2}$ (Tabla 1).

\section{Lóbulos Vagales}

Son estructuras pareadas de forma acorazonada tal como se puede observar en la figura 1 . En promedio equivalen al $7,70 \pm 0,59 \%$ de la longitud total y presentan un área promedio de $0,02 \pm 0,01 \mathrm{~cm}^{2}$ (Tabla 1).

\section{Lóbulos Faciales}

Al igual que los lóbulos vagales, son estructuras pareadas y acorazonadas. Son las estructuras más posteriores que se encuentran en sentido rostro-caudal del cerebro (Figuras 3 y 4). En promedio equivalen al 8,18 $\pm 0,80 \%$ de longitud total y poseen un área promedio de $0,03 \pm$ $0,01 \mathrm{~cm}^{2}$ (Tabla 1).

\section{Lóbulos Inferiores}

Tal como lo muestra la figura 4 estos lóbulos sólo pueden ser observados en la vista ventral del cerebro y son ovalados. Su longitud corresponde al 26,64 $\pm 1,67 \%$ y poseen un área promedio de $0,15 \pm 0,02 \mathrm{~cm}^{2}$ (Tabla 1).

\section{Discusión}

El encéfalo de A. seemanni ocupa la totalidad de la cavidad craneal, y a diferencia de otros peces como capitán de la sabana (Eremophilus muttisi) y neón cardenal (Paracheirodon axelrodi) no presenta tejido graso recubriéndolo en la parte dorsal (19). La presencia de este tejido está relacionada con el mantenimiento de la temperatura cerebral para peces que se desplazan a lo largo de la columna de agua o para aislamiento térmico en especies que habitan aguas frías como es el caso del capitán de la sabana (20). Teniendo en cuenta el conocimiento que se tiene acerca del hábitat de $A$. seemanni, se sabe que no tiene que enfrentar ninguna de las situaciones anteriormente planteadas, ya que habita en aguas cálidas que se encuentran entre $22-26^{\circ} \mathrm{C}$ y no tiene mucho desplazamiento verticalmente a lo largo de la columna de agua, de esta manera no enfrenta cambios de temperatura muy drásticos (3), para lo cual no se hace necesario que el cerebro se encuentre cubierto por tejido adiposo.

\section{Morfología y morfometría}

El tiburoncito presenta un patrón general de distribución de los varios lóbulos, característico de los teleósteos (13). Se han registrado varias relaciones entre los factores ambientales y el desarrollo del cerebro de los peces (21). Un estudio realizado en tres especies de ciprínido (Danio malabaricus, Rasbora argyrotaenia y Amblypharyngodon mola) concluyó que estas presentan gran variación intra e inter específica en el tamaño del encéfalo dependiendo del estilo de vida y el tipo de hábitat (22). Así mismo se han realizado investigaciones de teleósteos en donde se revela que la morfología del encéfalo se relaciona con parámetros comportamentales y ecológicos $(15,17,18)$. Un ejemplo de estas variaciones se hace evidente comparando la morfología del encéfalo de A. seemanni, la del capitán de la sabana (Eremophilus muttisi), ambos del orden de los siluriformes, y los cerebros del neón cardenal (Paracheirodon axelrodi) y la cachama blanca (Piaractus brachypoтиs), pertenecientes al orden characiformes, pues se observa que en los siluriformes los bulbos olfativos se encuentran conectados a los hemisferios telencefálicos a través de los tractos olfativos, contrario al caso de los characiformes en donde los bulbos olfativos se encuentran yuxtapuestos a los hemisferios. Esta diferencia podría indicar una mayor velocidad de llegada de los estímulos olfatorios a los lóbulos olfatorios de los characiformes, en comparación con los siluriformes.

Respecto al análisis morfométrico se obtuvo que la estructura más grande del encéfalo es el cerebelo (44 \pm $1,28 \%$; Área: $0,23 \pm 0,03 \mathrm{~cm}^{2}$ ), lo cual podría deberse a que esta estructura está encargada principalmente de la coordinación motora y, teniendo en cuenta que $A$. seemanni se caracteriza por ser un pez con tendencias carnívoras, la coordinación del movimiento para capturar la presa se hace importante, de la misma forma este pez es predado por otros de mayor tamaño y al momento de huir de predadores el movimiento debe ser altamente coordinado. Cadwallader (1975), (21) afirmó que en peces que habitan aguas rápidas, el cerebelo es de mayor proporción, debido a que el control del movimiento necesita ser más preciso que en aguas lentas. Lo anterior se ajusta a tiburoncito, teniendo en 
cuenta que este pez cambia de ambientes acuáticos y de velocidades de agua para desovar (3).

Los hemisferios telencefálicos también tienen un tamaño importante en relación con las demás estructuras $(28,15 \pm$ 0,57\%; Área: 0,13 $\pm 0,02 \mathrm{~cm}^{2}$ ), y según Huber et al 1997 (17) el tamaño de estas estructuras está relacionado con la habilidad de subsistir en ambientes espacialmente estructurados. Así mismo también está involucrado en aspectos como reproducción, visión a color y aprendizaje (23), lo que es importante en una especie cazadora como es el caso del tiburoncito. Se ha reportado que el telencefalo en goldfish, especialmente la parte dorso-lateral, está claramente relacionada al aprendizaje espacial y los procesos relacionados con la memoria (19). En tiburoncito esto se hace importante en la media que en los ambientes que acostumbra habitar esta especie el aprendizaje espacial debe ser fundamental para la sobrevivencia. Adicionalmente, esta especie se caracteriza por ser una especie territorialista y se ha reportado que en aspectos que incorporen criterios de comportamiento social como este, el desarrollo del los hemisferios telencefálicos se ve favorecido (17).

Los resultados de este estudio están de acuerdo con los presentados por Ari (2008) (24) quien sustentó que en general en Callorhinchus milii (Tiburón elefante), Squalus acanthias (Tiburón espinoso), Pristiophorus cirratus (Tiburón sierra trompudo), Cephaloscyllium laticeps (Pejegato australiano), Raja miraletus (Raya de espejos), Torpedo marmorata (Torpedo) y Dasyatis pastinaca (raya látigo común), de todos las estructuras del encéfalo, el telencéfalo y el cerebelo exhiben la mayor variación en tamaño y forma correspondiendo a las estructuras más grandes.

Con respecto al tamaño de los lóbulos olfativos en $A$. seemanni, se puede inferir que este sistema sensorial no es muy utilizado por la especie: A. seemanni habita la mayor parte de su vida en estuarios, donde no se presentan corrientes muy fuertes y se ha descrito que peces en estos hábitats presentan un modesto sentido del olfato y son dependientes de otro sistema sensorial para la ubicación del alimento (25). Caso contrario se presenta en especies que habitan grandes profundidades en donde se han encontrado bulbos y nervios olfativos muy grandes y desarrollados (14).

Los lóbulos vagales y faciales se encuentran desarrollados de manera importante en esta especie, en relación con especies pertenecientes al orden Characiformes. Esto se debe en parte a la presencia de barbillones ricos en receptores de señales cutáneas y gustativas $(14,15,18)$. Los siluriformes tienen sistemas gustativos muy elaborados, inervados por ramas del nervio facial, que terminan centralmente en el extremo rostral de la columna viscero-sensorial de la medula (26). Esto permite suponer que este es un sistema sensorial importante en la especie, así como se ha reportado en el género Mullus, en donde los barbillones tiene función táctil igual que a los órganos de la línea lateral (27).

Estudios realizados en cíclidos indican que la turbidez y la profundidad del agua influyen en la morfología del encéfalo, particularmente en el tamaño de las estructuras visuales como los ojos y el tectum óptico (17). Con base en lo anterior, y aunque $A$. seemanni no hace parte de los cíclidos, al habitar estuarios que poseen una turbidez considerable; posee un tamaño considerable de ojos respecto al tamaño de la cabeza.

Wanger, 2001 (28) reportó que el 40\% de las especies de peces utiliza primordialmente un sistema sensorial (especialistas), el $46 \%$ utiliza dos sistemas sensoriales (dominantes), y el 14\% restante actúa bajo tres sistemas sensoriales (generalistas). Teniendo en cuenta los resultados morfométricos obtenidos en este estudio, A. seemmanni podría considerarse un organismo que utiliza primordialmente la vista y el gusto, por lo cual podría ser clasificado como un dominante. Sin embargo, ya que los lóbulos ópticos son considerablemente más grandes que los centros gustativos, esta clasificación podría refinarse considerándolo un dominante con tendencia a la especialización visual.

Ahora bien, resaltando la importancia que tienen los barbillones para A. seemanni y su especialización visual, el tamaño de los lóbulos inferiores que presenta esta especie $\left(26,64 \pm 1,67 \%\right.$; Área: $\left.0,15 \pm 0,02 \mathrm{~cm}^{2}\right)$ puede estar relacionado, en la medida que estos reciben entradas visuales y gustativas, además que parecen estar involucrados con la percepción sensorial (8). La presencia de la línea lateral, la cual es considerada un órgano sensorial cutáneo está relacionada directamente con la crista cerebelaris la cual se encuentra situada caudal del cerebelo y muy probablemente tiene conexiones estructurales y también funcionales con este (24), lo cual, teniendo en cuenta la importancia anteriormente descrita del cerebelo en esta especie, podría dar razón del tamaño que presentan los lóbulos de la crista $\left(17,10 \pm 0,86 \%\right.$; Área: $\left.0,07 \pm 0,01 \mathrm{~cm}^{2}\right)$.

\section{Conclusiones}

La morfología del encéfalo de A. seemanni presentó el patrón general de teleósteos del orden Siluriforme, 
presentando el cerebelo como la estructura más grande del encéfalo y sugiriendo según la morfología y morfometría presentada que $A$. seemanni utiliza primordialmente la vista como el gusto para explotar su hábitat.

\section{Financiación}

Esta investigación se realizó gracias al apoyo económico del centro de Investigaciones de la Universidad Militar Nueva Granada. Proyecto de Investigación Científica CIAS No 428.

\section{Conflictos de intereses}

Los autores declaran no tener conflictos de intereses.

\section{Referencias}

1. Pinheiro-Tavares MO. Suribim (The suribim catfish). En Surubim. Serie estudios Pesca N 19. Ministerio do medio ambiente, dos recursos hídricos e da Amazonia Legal. Instituto Brasileiro do Medio Ambiente e do Recursos Naturais Renováveis (IBAMA). Belo Horizonte 1997; 1-25.

2. Froese R, Pauly D FishBase. www.fishbase.org versión (12/2008). Consultado el 10 de febrero de 2009.

3. Nelson JS. Fishes of the world. Tercera edición. John Wiley y Sons, Inc., New York. 1994, 600p.

4. Lisney T, Bennett M, Colin S. Volumetric analysis of sensory brain areas indicates ontogenetic shifts in the relative importance of sensory system in elasmobranchs. Academia Sinica and National University of Singapore. The Raffles Bulletin of Zoology 2007; 14: $7-5$.

5. Kihslinger R, Nevitt G. Early rearing environment impacts cerebellar growth in juvenile salmon. Section Neurobiology Physiology and Behavior, UC Davis, Davis CA, USA. The Journal of Experimental Biology 2005; 209: 504-509.

6. Tovar MO, Contreras LF, Caldas ML, Rodriguez D, Hurtado H. Comparación histológica y morfométrica entre el ojo de capitán de la sabana (Eremophilus mutisii) y el ojo de trucha arco iris (Oncorhynchus mykiss). Acta Biológica Colombiana 2008; 13: 7378.

7. Roberts RJ. Fish pathology. Tercera edición. Edt. W.B Saunders. Toronto. 2001, 46p.

8. Butler A. Nervous System. En: Ostrander, G., Hopkins, J. y Baltimorie, M. D. The Laboratory Fish. Part 3:
Gross Functional Anatomy. USA.Academic Press. 2000, 331-345p.

9. Cannatella D. Central Nervous System. Brain and CNS. Fall. 2001, 1-5p.

10. Ann BB, William H. Comparative vertébrate neuroanatomy. Wiley-liss. United States of American. 1996, 77-88p.

11. CCAC guidelines on: the care and use of fish in research, teaching and testing. 2003.

12. Ishikawa Y, Yoshimoto M, Yamamoto N, Ito H. Different brain morphologies from different genotypes in a single teleost species, the medaka (Oryzias latipes) Brain. Behaviour and Evolution; 1999a; 53 (2): 74.

13. Mukuda T, Ando M. Brain atlas of the Japanese Eel: comparison to other fishes. Memories of Faculty of Integrated Arts and Science., Hiroshima University 2003; 29: 1-25.

14. Evans H. Anatomy, Genetics and Breeding, Anatomy of tropical Fishes. En: Gratzek J. y Matthews J. Aquarology: The science of fish health mamagement. Editorial: Tetra Press. 1992, 77-78, 81-83, 92-93p.

15. Kotrschal K, Van Staaden M, Huber R. Fish brains: evolution and environmental relationships. Reviews in Fish Biology and Fisheries 1998; 8: 373-408.

16. Marchetti M, Nevitt G. Effects of hatchery rearing on brain structures of rainbow trout, Oncorhynchus mykiss. Environmental Biology of Fishes 2003; 66: 9-14.

17. Huber R, Van Staaden M, Kaufman L, Karel F. Microhabitat Use, Trophic Patterns, and the Evolution of Brain Structure in African Cichlids. KARGER. Brain, Behaviour and Evolution 1997; 50: 167-182.

18. Van Staaden M, Huber R, Kaufman L, Karel F. Brain evolution in cichlids of the African Great Lakes: brain and body size, general patterns, and evolution trends. Zoology 1995; 98: 165-178.

19. Gómez Y, Vargas J, Portavella M, López J. Spatial learning and goldfish telencephalon NMDA receptors. Neurobiology of Learning and Memory 2006; 85: 252-262.

20. Carey N. A brain heater in the Swordfish. Science 1982; 216 (4552): 1327-1329.

21. Cadwallader P. Relationship between brain morphology and ecology in New Zealand Galaxidae, particularly Galaxias vulgaris (Pisces: Salomoniformes). Department of Zoology, University of Canterbury, Christchurch, New Zealand. New Zealand Journal of Zoology 1975; 2 (1): 35-43. 
22. Balescu C. A comparative study on the external morphology of the encephalon in three fish species belonging to Cyprinidae family: Danio malabaricus, Rasbora argyrotaenia and Amblypharyngodon mola. Analele Stiintifice ale Universitatii 2005:45-50.

23. Salas C, Broglio C, Durán E, Gómez A, Ocaña FM, Jiménez-Moña F, Rodríguez F. Neuropsychology of Learning and Memory in Teleost Fish Zebrafish 2006; 3 (2): 157-171.

24. Ari Csilla. Correlation between the cerebralization, astroglial architecture and blood-brain barrier composition in Chondrichthyes. Tesis Doctoral. Semmelweis University Neurobiology School of Doctoral Studies, Budapest, 2008, 130 p.
25. Chiason R, Radke W. Laboratory anatomy of the perch. Cuarta edición. WCB McGraw-Hill. 1991, Cap: 10. 68-79p.

26. Kiyohara S, Sakata Y, Yoshitomi T, Tsukuhara J. The "goatee" of goatfish: innervation of taste buds in the barbels and their representation in the brain. Proceedings Biological Sciences 2002; 269 (1502): 1773-1780.

27. Roule M, Verrier M. Etude sur les barbillons des Rougets-barbest (G. Mullus). Annales de la Station Océanographique de Salammbô 1927; 3: 1-16.

28. Wanger H. Brain areas in abyssal demersal fishes. Brain, Behaviour and Evolution. 2001; 57: 301-316. 